This item was submitted to Loughborough's Research Repository by the author.

Items in Figshare are protected by copyright, with all rights reserved, unless otherwise indicated.

\title{
Temporal relations among multidimensional perceptions of competence and trichotomous achievement goals in physical education
}

PLEASE CITE THE PUBLISHED VERSION

http://dx.doi.org/10.1016/j.psychsport.2011.04.007

PUBLISHER

(C) Elsevier Ltd

VERSION

AM (Accepted Manuscript)

LICENCE

CC BY-NC-ND 4.0

\section{REPOSITORY RECORD}

Spray, Christopher M., and Victoria E. Warburton. 2019. "Temporal Relations Among Multidimensional Perceptions of Competence and Trichotomous Achievement Goals in Physical Education”. figshare. https://hdl.handle.net/2134/11346. 
This item was submitted to Loughborough's Institutional Repository (https://dspace.lboro.ac.uk/) by the author and is made available under the following Creative Commons Licence conditions.

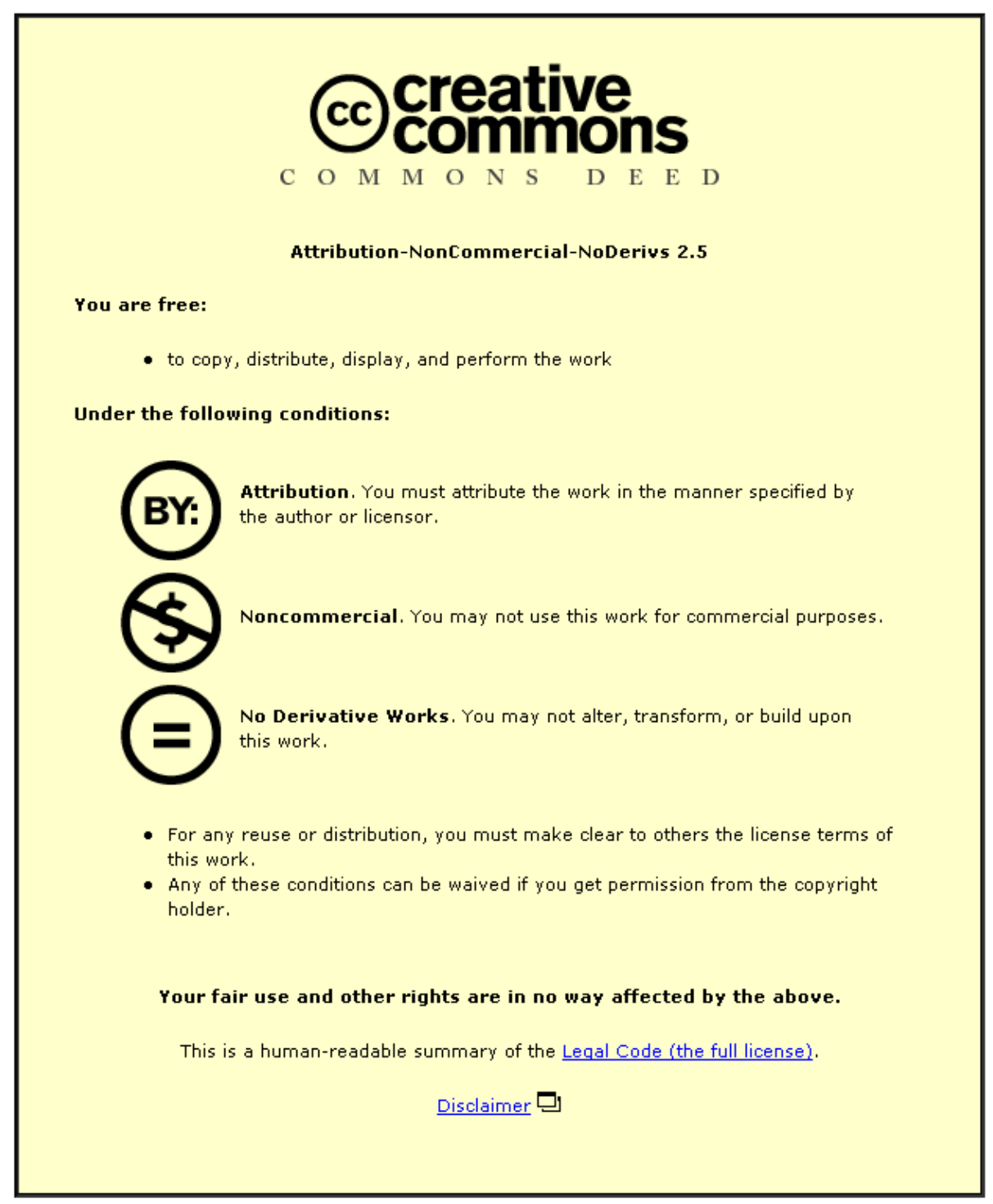

For the full text of this licence, please go to: http://creativecommons.org/licenses/by-nc-nd/2.5/ 
1

2

3

4

5

6

7

8

9

10

11

12

13

14

15

16

17

18

19

20
Temporal Relations Among Multidimensional Perceptions of Competence and Trichotomous Achievement Goals in Physical Education

Date submitted: August $4^{\text {th }}, 2010$

Date resubmitted: November $24^{\text {th }}, 2010$

Date of third submission: February $24^{\text {th }}, 2011$

Date of fourth submission: April 20 ${ }^{\text {th }}, 2011$ 


\section{Abstract}

2 Objectives: The purpose of the present study was two-fold: (1) To empirically establish

3 whether young people differentiate their perceived competence in physical education (PE) in

4 terms of the self, mastery of tasks, and others, and (2) To examine longitudinal relations

5 between these three ways of defining perceived competence and trichotomous achievement

6 goals.

7 Methods: At the start of the study, students $(n=227$ males, $n=205$ females; $M$ age $=13.18$,

$8 S D=0.87$ years) completed measures of mastery-approach, performance-approach and

9 performance-avoidance goals, along with other-, self- and mastery-referent forms of perceived competence. The same measures were subsequently recorded three, six and nine months later.

11 Results: Analyses supported longitudinal factorial invariance for each goal and each type of 12 perceived competence. Partial support was found for the positive influence of other-referent perceived competence on approach and avoidance performance goal adoption over time.

14 Conclusion: Young people can construe their competence in PE in various ways. Relative to one’s classmates, increases in other-referenced perceptions of competence can subsequently

16 lead to increased adoption of both performance goals.

Keywords: approach-avoidance achievement goals, perceived competence, physical education 
Temporal Relations Among Multidimensional Perceptions of Competence and Trichotomous Achievement Goals in Physical Education

Recent theorizing has proposed that competence should serve as the conceptual centerpiece of research into achievement motivation. Assigning competence a core role will, according to Elliot and Dweck (2005), help to bring clarity and parsimony to the achievement motivation literature because competence can be defined and operationalized in precise ways. A number of different theories of achievement motivation have incorporated the competence concept, including achievement motive and attribution frameworks. One perspective that has received a great deal of empirical attention during the past twenty-five years, in both education and physical domains, is achievement goal theory (Dweck, 1986; Elliot, 1997, 1999; Nicholls, 1984, 1989). Recently, Elliot and co-workers have sought to clarify the conceptualization of competence within the achievement goal framework, as well as to propose the nature of the relationships between competence and goals (see Elliot, 1999, 2005; Elliot \& Church, 1997; Elliot \& Harackiewicz, 1996). However, limited empirical attention has focused on the interrelationships between goals and competence in the physical domain using this contemporary perspective. Moreover, the extant body of literature has largely failed to take into account the more precise ways in which competence may be defined when testing associations with goals. In particular, from a developmental perspective, we know little about the transactional nature of relations and whether bidirectional relationships exist (Sameroff, 2009).

The present study tested the direction and magnitude of relations between young people's perceived competence and goal striving in school physical education (PE) over time. In line with contemporary theory (Elliot, 2005), relations were examined between goals and more nuanced definitions of competence.

\section{Perceptions of Competence and Trichotomous Achievement Goals}


The work of Elliot and associates adopts a motivational analysis of competence and

2 therefore examines how competence energizes and directs individuals' behavior in settings

3 where competence is salient. Competence is viewed as a basic fundamental psychological need

4 that activates behavior (Elliot, McGregor, \& Thrash, 2002). However, as a consequence of

5 experience and socialization, individuals develop the need not just to develop or demonstrate

6 competence but to avoid developing or displaying incompetence. Importantly, Elliot and his

7 colleagues (Elliot, 1999, 2005; Elliot \& McGregor, 2001; Elliot et al., 2002) distinguish three

8 standards of competence that individuals may use in evaluating performance: "absolute (the

9 requirements of the task itself), intrapersonal (one’s own past attainment or maximum potential

attainment), and normative (the performance of others). That is, competence may be evaluated,

and therefore defined, according to whether one has acquired understanding or mastered a task,

improved one’s performance or fully developed one’s knowledge or skills, or performed better than others” (Elliot \& McGregor, 2001, p.501). Achievement goal research in the domain of sport and physical activity has ignored these separate standards by which competence can be defined, although researchers have occasionally incorporated these distinct facets within measures of goal attainment. That is, attainment can be judged in terms of whether individuals perceive task mastery, self-improvement or superiority over others (Amiot, Gaudreau, \& Blanchard, 2004; Soucy Chartier, Goudreau, \& Fecteau, 2011). Assessment of individuals’ level of perceived competence per se has combined self-referent and norm-referent items within the same measure or has focussed exclusively on normative items. Moreover, commonly used items and response scales have been vague with respect to the definition of competence (e.g., “How good are you at ....?”; “Not at all good - Very good”). Consequently, relationships between specific types of competence perceptions and goals remain poorly understood. In the trichotomous achievement goal framework, three achievement goals are posited 
1 desirable outcomes or striving to avoid aversive events and possibilities (Elliot, 1999). Hence,

2 goals represent the aims of individuals' behavior and these approach- and avoidance-oriented

3 aims emerge, in part, from perceptions of competence (Elliot \& Church, 1997; Elliot \& Thrash,

4 2001). A mastery-approach (MAp) goal focuses on developing self- and mastery-referent

5 competence, a performance-approach (PAp) goal focuses on demonstrating norm-referent

6 competence, and a performance-avoidance (PAv) goal focuses on avoiding demonstrating

7 normative incompetence. Examples in the physical domain include: trying to improve one’s

8100 metre freestyle time (MAp); trying to beat an opponent in badminton (PAp); and striving to

9 avoid finishing last in a football tournament (PAv).

\section{Relations Between Perceived Competence and Achievement Goals}

$$
\text { Competence perceptions are conceptualized by Elliot and colleagues to directly }
$$

determine adoption of goals. Approach goals are theorized to emerge from higher perceptions of competence, whereas lower perceptions of one's competence are posited to bring about the adoption of avoidance goals (Elliot, 1999; Elliot \& Church, 1997). In PE, individuals with high perceived competence are likely to have received positive feedback and praise for their efforts and achievements from their teachers and peers, and thus may be more likely to seek further improvement and normative success. On the other hand, those individuals for whom criticism and embarrassment have led to low perceptions of competence are more likely to seek to avoid further negative outcomes and comparisons in PE classes. Although support for proposed relationships has been found in the educational domain (e.g., Elliot \& Church, 1997), research in the physical domain has yielded mixed findings (e.g., Morris \& Kavussanu, 2008; Stevenson \& Lochbaum, 2008; Warburton \& Spray, 2008). Perceived competence has been positively associated with PAv goals as well as PAp goals, suggesting that individuals who report confidence in their abilities nevertheless strive to avoid normative failure because, in so doing, they are more likely to increase their chance of success (see Covington, 1992). 
In terms of the physical domain, achievement goal researchers have also posited paths

2 from performance goals to perceived competence (e.g., Goudas, Biddle, \& Fox, 1994),

3 suggesting a direction of influence from goals to perceived competence. This direction of

4 influence stands in contrast to the framework proposed by Elliot and co-workers which clearly

5 views perceived competence to determine goals. Nevertheless, it is plausible that the aim of

6 individuals' behavior affects how they feel about their competence. For example, pursuing

$7 \quad$ MAp goals, with their emphasis on absorption in the task and high effort, may result in

8 enhanced self-referent competence. Consequently, researchers should seek to clarify whether

9 competence underpins goals, whether goals underpin competence, or whether bidirectional

effects occur. In order to achieve this aim, studies need to incorporate at least two measurement waves.

To date, however, studies of competence perceptions and goals in the physical domain have overwhelmingly adopted a cross-sectional design (for a review, see Biddle, Wang, Kavussanu, \& Spray, 2003). We know little, for example, about the stability or continuity of competence perceptions and goals over time in different physical contexts and whether change in one construct impacts on change in another construct. That is, the transactional nature of the relationships between goals and competence has not received attention. For example, does change in one's perceived normative competence predict change in the adoption of PAp goals at a subsequent time point or vice-versa? Is full cross-prediction in evidence, whereby residual change in perceived competence and goals predicts subsequent residual change in goals and perceived competence respectively? Depending on the time interval of interest, PE students can encounter several compulsory activities with different classmates and teachers across occasions of measurement. The PE context, therefore, represents a unique physical setting in which to examine motivational phenomena among young people over time.

\section{The Present Study}


The present investigation sought to examine temporal relations between perceived

2 competence and trichotomous achievement goals within the context of school PE. In line with

3 Elliot's (1999, 2005) multidimensional conceptualization of competence, our first aim, utilizing

4 confirmatory factor analytic procedures, was to determine students’ competence perceptions

5 from three standards: self-referent (intrapersonal), mastery-referent (absolute), and other-

6 referent (normative). Given acceptable factorial invariance of the different types of perceived

7 competence over time, our second aim was to assess the relationships between the three types

8 of perceived competence and the three goals across four waves of measurement.

We anticipated that temporal patterns of stability and change would differ across types of perceived competence and goals. When students change curriculum activity, the new activity represents an opportunity to develop self- and mastery-referent competence to a lesser or

12 greater extent. In addition, it is possible that the perceived normative ability of class members changes due to factors such as previous experience and rate of learning, leading to variability in normative competence scores across activities. Similarly, different activities may promote the adoption of particular goals (e.g., overtly competitive team games versus typically more individualistic activities such as gymnastics and health and fitness). Given that, within Elliot's

17 framework, competence perceptions represent one antecedent among an array of potential antecedents that differentially relate to achievement goal adoption, we expected relations between perceived competence and goals to be moderate in magnitude (Elliot, 1999, 2005). In

20 accordance with theory and research, we also hypothesized that perceptions of competence

21 would be positively associated with approach goals (Elliot, 1999, 2005; Elliot \& Church, 22 1997). 


\section{Method}

\section{Participants}

At the first measurement wave, 432 students (male $n=227$, female $n=205$ ) from PE classes in Years 7, 8 and 9 of a state comprehensive high school in East England, United Kingdom, took part in the research. Participants were aged between 11 and 15 years $(M=$ $13.18, S D=0.87$ years) at wave 1 . The socio-economic circumstances of the students that attended the school were below the national average. Less than $5 \%$ of students came from minority ethnic backgrounds or spoke English as an additional language (Office for Standards in Education, 2007, p. 3). A team of two female and two male teachers taught compulsory PE classes, each typically comprising 30 students.

\section{Procedures}

Ethical approval for the research procedures, which complied with the guidelines of the British Psychological Society, was obtained from the relevant institutional body. Permission for conducting the research was sought from the head teacher and head of physical education at the school. Parental consent was obtained through distribution of letters prior to data collection. Following an introduction to the purpose of the research, informed assent was given by participants through the completion of a willingness to participate form. All parents provided consent and no student refused to take part or asked for their data to be subsequently withdrawn. All procedures took place prior to a normal curriculum PE lesson. Participants were given an explanation of how to complete each section of the questionnaire and were provided with the opportunity to ask any questions. All participants were assured that the information collected would remain confidential and would have no effect on their PE report. Each participant responded anonymously to the questionnaire which took approximately 20 minutes to complete. However, knowledge of each participant's class/teacher, date of birth and gender 
1 allowed for matching of responses at later measurement waves. The questionnaires were

2 counterbalanced prior to distribution. The first wave of data collection took place in April and

3 all procedures were repeated in July of the same school year and again in October and January

4 of the following academic year.

\section{Measures}

Each participant completed a questionnaire that collected the following information.

Personal details. Three items relating to form group, date of birth, and gender comprised this section of the questionnaire.

Goal adoption. Goal adoption was assessed using three sub-scales from the Achievement Goal Questionnaire for Sport (AGQ-S, Conroy, Elliot, \& Hofer, 2003). The individual item stem of 'In PE/Sport what are your main concerns?' preceded the items. The nine items were answered on a seven-point Likert scale that ranged from not at all true of me (1) to very much like me (7). Three items assessed each type of goal. Sample items included, 'It is important for me to perform as well as I possibly can' (MAp), 'It is important to me to do well compared to others' (PAp), 'I just want to avoid performing worse than others' (PAv). The fourth sub-scale of mastery-avoidance (MAv) was not utilized in the present study owing to the conceptual and empirical limitations of the AGQ-S items (for a full discussion, see Elliot \& Murayama, 2008). Support for the factor structure of the AGQ-S and the reliability and validity of the MAp, PAp and PAv goals has been established (Conroy, Elliot, et al., 2003).

Perceived competence. This was assessed using six items answered on a five-point Likert scale that ranged from strongly disagree (1) to strongly agree (5). Two items assessed perceived competence (PC) relating to mastery of the task (PC Mastery) ('I am often able to successfully complete the activities I am set in PE', 'The activities that my teacher sets in PE I can usually do well'). Two items assessed PC relating to the self (PC Self) ('I can perform tasks and skills 
1 in PE better than I used to', 'I am better at activities in PE than I used to be') and two items

2 assessed PC relating to others (PC Other) ('I am better at PE than others in my class', 'I am one

3 of the best at PE in my class').

\section{Data Analysis}

All analyses were carried out using EQS 6.1 software (Bentler \& Wu, 2002). Logistic regression analyses revealed that the pattern of missing data was not significantly associated with participants' perceived competence or achievement goal scores at the start of the study. ${ }^{1}$ All analyses employed full information maximum likelihood estimation techniques. Given that the normalized estimate of Mardia's coefficient of multivariate kurtosis was high (lowest value for achievement goals was 29.91 and for PC 12.37), the robust maximum likelihood estimation procedure was used.

Preliminary analyses: Factorial validity and longitudinal factorial invariance. In order to assess the factorial validity of the new measure of perceived competence, a series of nested models testing alternative structures was analysed across time. These analyses assessed whether the a priori factor structure was evident at each wave of measurement. Specifically, four alternative models were examined; a unidimensional (one factor), dichotomous (two factors: self/mastery and other), trichotomous (three factors: self, mastery, and other), and hierarchical model (four factors: a second-order PC factor underpinned by self, mastery, and other firstorder factors). In addition, the factor structure of goals, as assessed by the AGQ-S, was examined across time.

Following confirmatory factor analysis (CFA), the longitudinal factorial invariance (LFI) of achievement goals and types of PC was assessed using six separate models, one for each achievement goal (MAp, Pap, and PAv), and one for each type of PC (other, self, and mastery). In line with research conducted in the physical domain, the LFI of the individual models was assessed using a series of nested models with progressively more constrained 
1 model parameters (see Conroy, Elliot, et al., 2003; Conroy, Kaye, \& Coatsworth, 2006;

2 Conroy, Metzler, \& Hofer, 2003).

Main analyses: Temporal relations between perceived competence and achievement goals. A

series of structural equation models was examined to test the temporal relations between the three types of PC and each of the three achievement goals. The procedures for these analyses were informed by developmental theory and empirical research in the physical domain (Conroy \& Elliot, 2004; Sameroff, 2009). Specifically, we tested separate models for each achievement goal to reduce the complexity of the results and because the scales may be used independently of each other in research settings (Conroy, Elliot et al., 2003). Corresponding item uniquenesses across waves of measurement and the latent factors at wave one were allowed to covary. Factor loadings and item uniquenesses were constrained to be invariant over time, however, the covariance of the within-time factor disturbances was freely estimated at waves two, three and four. Four nested models were specified (see Figure 1). 1) The no cross prediction (stability) model established paths only between each latent variable and the subsequent corresponding latent variable (i.e., all cross-lagged paths were fixed to zero); 2) In addition to the paths of the no cross prediction model, the second model added directional paths from PC to achievement goals (e.g., self-referent PC at time 2 to MAp goals at time 3). This model was labelled the PC-Goals model; 3) In addition to the paths of the no cross prediction model, the third model, referred to as the Goals-PC model, added directional paths from achievement goals to PC (e.g., PAp goals at time 3 to other-referent PC at time 4); 4) In the final model, paths were established between each latent variable and the subsequent corresponding latent variable and from early PC or goals to subsequent goals and PC respectively (i.e., all cross-lagged paths were freely estimated). This model was referred to as the full cross prediction model and served as the baseline model for model comparisons as it estimated the most non-zero paths. In the testing of the temporal relations between PC and 
1 achievement goals, if the PC-Goals or Goals-PC models were revealed to be plausible

2 alternatives based on comparison of model fit with the full cross prediction model, they were

3 then examined against the no cross prediction model. This final model comparison examined

4 whether any additional constraints (i.e., fixing the cross-lagged paths to zero) could be imposed

5 on the model without significant loss of fit (cf. Conroy \& Elliot, 2004).

\section{INSERT FIGURE 1 HERE}

Evaluation of model fit. In all analyses, model fit was evaluated using comparisons of absolute (chi-square, consistent version of Akaike’s information criterion [CAIC], root mean square error of approximation, [RMSEA], standardized root mean square residual, [SRMR]) and relative (non-normed fit index, [NNFI], comparative fit index [CFI]) fit indices. In line with Hu and Bentler's (1999) recommendations, values of $\geq 0.90$ and $\geq 0.95$ for the NNFI and CFI were taken as representing an acceptable and good fit to the data respectively. Values of $\leq 0.6$ and $\leq$ 0.8 were taken as indicating good model fit for the RMSEA and SRMR respectively. Scaled chi-square difference tests were also used in conjunction with these fit indices to assess which of the nested models showed better fit to the data (Satorra \& Bentler, 2001).

\section{Results}

Descriptive statistics and internal consistency estimates for each of the three achievement goals and types of PC are presented in Table 1. Factor correlations between all study variables across all measurement waves are shown in Table 2. Each scale exhibited acceptable internal consistency in that Cronbach's alpha exceeded the 0.70 criterion at each wave of measurement. Correlations among the three types of perceived competence were less than unity (range $=.40$ to .82), supporting the notion of distinct but related definitions of competence. Correlations among the three achievement goals ranged from .55 to .95 . In particular, both performance goals were strongly and positively correlated at each time point. 
1 Preliminary Analyses: Factorial Validity and Longitudinal Invariance

2 Perceived competence. The CFA results for the PC questionnaire indicated that the

3 hypothesized factor structure, three separate but correlated factors, exhibited a good fit to the

4 data across all measurement occasions $\left[\mathrm{SB} \chi^{2}(186)=197.56, p>.05\right.$; NNFI = .994; CFI = .996;

$5 \quad \operatorname{SRMR}=.031$; RMSEA $(90 \% \mathrm{CI})=.015(.000-.031)]$. Relative and absolute fit indices were

6 superior to alternative factor structures. The dichotomous model of PC revealed acceptable

7 goodness of fit, but scaled chi-square difference test showed this model to be inferior to the

8 trichotomous model. The one PC factor model and the hierarchical model showed poor fit. In

9 the three factor model, all factor loadings exceeded .68 and each item loaded significantly on

10 its hypothesized latent variable at all measurement occasions.

11 The LFI analyses to assess the structural stability and invariance of responses to the three sub-

12 scales of the PC questionnaire showed that according to the absolute fit criteria, PC Mastery

13 achieved weak factorial invariance whereas PC Other and PC Self achieved strong factorial

14 invariance. However, each PC factor achieved strict factorial invariance with respect to relative

15 fit indices. In summary, the CFA analyses revealed that all further analyses should utilize the

16 three factor model of PC (i.e., PC Other, PC Self and PC Mastery) while the LFI analyses

17 revealed that the three types of PC exhibited acceptable longitudinal factorial invariance

18 (Meredith \& Horn, 2001). ${ }^{2}$

Achievement goals. Given the higher inter-factor correlations among the two performance goals over time, the fit indices of a series of nested models were examined. The CFA results

21 revealed that the a priori factor structure of the trichotomous achievement goal framework, that 22 is three separate but correlated goals, exhibited a good fit to the data across measurement waves $23\left[\mathrm{SB} \chi^{2}(528)=689.88, p<.001 ; \mathrm{NNFI}=.956 ; \mathrm{CFI}=.963 ;\right.$ SRMR $=.050 ;$ RMSEA $(90 \% \mathrm{CI})=$ 24.034 (.026-.040)]. Relative and absolute fit indices were superior to alternative factor structures 25 (e.g., PAp and PAv items comprising one factor along with a separate MAp factor; 
1 trichotomous model with the correlation between performance goals constrained to 1 ). In the

2 trichotomous model, all factor loadings exceeded .61 and each item loaded significantly on its

3 hypothesized latent variable at each wave of measurement.

The LFI analyses to assess the structural stability and invariance of responses to the three achievement goal sub-scales showed that according to the absolute fit criteria, the MAp and PAv factors achieved weak factorial invariance and the PAp factor achieved strong factorial invariance. However, due to the influence of sample size on these indices, it is also necessary to assess the factorial invariance of the models in terms of the relative fit criteria (Marsh, Balla, \& McDonald, 1988). Each achievement goal model achieved strict factorial invariance with respect to relative fit indices. In summary, these analyses revealed that the three achievement goals exhibited acceptable longitudinal factorial invariance (Meredith \& Horn, 2001) ${ }^{3}$

Main Analyses: Temporal Relations between Perceived Competence and Achievement

\section{Goals}

The temporal relations among the three types of PC and the three achievement goals were examined using a series of structural equation models. Tables 3-5 show the absolute and relative fit indices and the nested model comparisons for each type of PC with each of the three achievement goals.

PC and MAp goals. Table 3 shows that models for PC Mastery and PC Self exhibited acceptable to good fit to the data, whereas the models for PC Other and MAp goals generally did not show as good fit indices (notably model CAIC, NNFI and RMSEA). The nested model comparisons showed that model fit did not significantly decrease when paths which originate in early PC (Self or Mastery) or MAp goals and predict subsequent MAp goals or PC (Self or Mastery) respectively were constrained to zero. There was also no significant decrease in model fit when comparing the PC-Goals and Goals-PC models with the no cross prediction 
model. It was therefore concluded that the no cross prediction model (i.e., non-significant prospective paths) was more parsimonious and was the best model for PC Self/Mastery and MAp goals.

\section{INSERT TABLE 3 HERE}

PC and PAp goals. Table 4 shows that each model exhibited a good fit to the data for each type of PC with PAp goals. The nested model comparisons for PC Other with PAp goals showed that model fit decreased significantly with the removal of paths which originate in early PC Other and predict subsequent PAp goals, but not with the removal of paths which originate in early PAp goals and predict subsequent PC Other. It was therefore concluded that for PC Other and PAp goals, the best model was the PC-Goals model, whereby paths originating in early PC Other predict subsequent PAp goals. The standardized parameter estimates for PC Other and PAp goals are presented in Figure 2 and show that individuals with positive change scores in PC Other at Time 2 increased adoption of PAp goals at Time 3.

For PC Self and PC Mastery and PAp goals, the nested model comparisons showed that model fit did not significantly decrease when paths which originate in early PC (Self and Mastery) or PAp goals and predict subsequent PAp goals or PC (Self and Mastery) respectively were constrained to zero. There was also no significant decrease in model fit when comparing the PC-Goals and Goals-PC models with the no cross prediction model. It was therefore concluded that the no cross prediction model (i.e., non-significant prospective paths) was more parsimonious and was the best model.

\section{INSERT TABLE 4 AND FIGURE 2 HERE}

PC and PAv goals. Each model exhibited a good fit to the data for each type of PC with PAv goals (see Table 5). The nested model comparisons for PC Other with PAv goals showed that model fit decreased significantly with the removal of paths which originate in early PC Other and predict subsequent PAv goals, but not with the removal of paths which originate in early 
1 PAv goals and predict subsequent PC Other. It was therefore concluded that for PC Other with

2 PAv goals, the best model was the PC-Goals model, whereby paths originating in early PC

3 Other predict subsequent PAv goals. The standardized parameter estimates for PC Other and

$4 \quad$ PAv goals are presented in Figure 3 and show that individuals with positive change scores in

$5 \quad$ PC Other at Time 2 increased adoption of PAv goals at Time 3.

Finally, for PC Self and PC Mastery and PAv goals, the nested model comparisons showed that model fit did not significantly decrease when paths which originate in early PC (Self and Mastery) or PAv goals and predict subsequent PAv goals or PC (Self and Mastery) respectively were constrained to zero. There was also no significant difference in model fit when comparing the PC-Goals and Goals-PC models with the no cross prediction model. It was therefore concluded that the no cross prediction model (i.e., non-significant prospective paths) was more parsimonious and was the best model.

\section{INSERT TABLE 5 AND FIGURE 3 HERE}

In sum, these results indicated differential temporal patterns among types of perceived competence and the three achievement goals of the trichotomous goal framework. Findings for PAp and PAv goals were similar in that PC Other was shown to be a moderately strong predictor of both goals, whereas the no cross prediction model was the best fitting for both PC Mastery and PC Self and the three goals.

\section{Discussion}

The present study sought to examine temporal relations between different types of

21 perceived competence and approach-avoidance achievement goals within the context of school PE. More specifically, we first set out to establish support for the factorial validity of three types of perceived competence: self-improvement in PE activities, capacity to fulfil/master tasks set by the teacher, and normative comparisons of ability with classmates. Our second aim was to assess the magnitude and direction of relationships among these different competence 
1 perceptions and the achievement goals across four time points spanning a period of nine

2 months.

The present research provides initial insight into young people’s capacity to distinguish different types of perceived competence in the PE context, providing support for recent theorizing (Elliot, 1999, 2005; Elliot \& Dweck, 2005). Each definition of competence exhibited acceptable internal consistency and strict factorial invariance across measurement occasions. Previous research in the physical domain has typically examined perceived competence as a unidimensional factor. In the current study, the three-factor model demonstrated superior fit indices when compared with alternative models. We suggest that identifying more nuanced perceptions of competence will further knowledge of the motivational consequences for individuals holding high or low perceptions. With regard to the present investigation, we examined the effects of changes in students' specific competence perceptions on changes in goal adoption in PE (Elliot, 1999, 2005). However, in line with research and theory (e.g., Goudas et al., 1994; Sameroff, 2009), we tested alternative patterns of temporal relations. Continuity was demonstrated for the three types of perceived competence and the three achievement goals across all occasions of measurement. These continuities reflect rank-order or relative stability in the corresponding constructs. However, PE represents a unique setting in which young people pursue a range of different physical activities over time. Consequently, we expected to see inter-individual differences in competence perceptions and goals as students encountered new activities in differing environments.

There was some support for the theoretical proposition (Elliot, 1997, 1999; Elliot \& Church, 1997; Elliot \& Harackiewicz, 1996) that perceived competence influences the adoption of goals. The PC-goals model was shown to be the better fitting model for PC Other with PAp goals and for PC Other with PAv goals. Students who increased their rank-order position in terms of perceptions of normative competence also increased their relative position in terms of 
1 both types of performance goals between the second and third measurement waves. However,

2 this relationship was not found between the third and fourth waves, which could be a result of

3 the change of PE activities during this time frame. In future, studies need to consider more

4 explicitly the influence of changing PE activities on the self-perceptions and goals of young

5 people. It was also apparent that, across waves two and three, students who reported greater

6 increases in perceptions of norm-referenced competence relative to their classmates also

7 reported greater adoption of goals focussed on avoiding being incompetent compared to others.

8 This relationship is counter to the theoretical propositions of Elliot and co-workers, and may be

9 explained by the high correlation between the performance goals at all time points.

Consequently, the finding of a positive relationship between normative perceived competence and PAv goals should be viewed with caution, however, it is consistent with emerging evidence in education and PE contexts (Jagacinski, Kumar, Boe, Lam, \& Miller, 2010; Wang, Biddle, \& Elliot, 2007; Warburton \& Spray, 2008). The positive influence of normative perceived competence on PAv goals may also be a consequence of the perception of classroom and PE settings as particularly performance- or ego-involving (i.e., characterized by evaluation of ability and greater recognition and attention given to the normatively more able; Ames, 1992).

Thus, further work is warranted on the influence of the context on the relationship between normative perceived competence and the adoption of PAv goals (Jagacinski et al., 2010). The present study found little support for higher perceptions of competence predicting increased adoption of MAp goals. When examining MAp goals, the no cross-prediction model was the preferred model for both PC Self and PC Mastery. Other antecedents may moderate the relationship of perceived competence with MAp goals. One candidate is likely to be implicit theories of athletic ability (Biddle, Wang, Chatzisarantis, \& Spray, 2003; Dweck, 1999). Researchers should examine the proposition that high perceived competence in conjunction 
1 with a view of PE ability as acquirable and increasable is more likely to facilitate the adoption

2 of MAp goals than high perceived competence combined with a fixed, entity view of ability.

\section{Limitations, Future Directions, and Conclusions}

The current study provides an initial attempt to examine the dynamic relationships among constructs drawn from contemporary achievement goal theory, using a transactional perspective to identify the influence of ‘change on change’ (Sameroff, 2009). We identified specific time periods when increased perceptions of normative competence predicted increased adoption of PAp and PAv goals. Such findings are important because they clarify the direction of influence whereby the predictor variable clearly precedes the criterion variable. However, noting that relative increases in perceived competence reported at one moment in time lead to relative increases in reported goal adoption three months later takes no account of the numerous 'transactions' between perceived competence and goals that occur during the period of time i.e., perceived competence may change between waves two and three and thus exert a withintime influence on goal adoption (and vice-versa) (see Gershoff, Aber, \& Clements, 2009). In the present study, moderate to strong correlations were found between types of perceived competence and goals at all measurement waves, indicating significant contemporaneous relationships among the constructs. Fixing these within-time parameters to zero resulted in considerably worse model fit in all cases. Developmental research in sport and exercise psychology should utilize continuous or nonrecursive models to identify the magnitude and direction of influence of these within-time relationships.

Moreover, researchers conducting longitudinal studies need to consider the time interval between measurement waves, as longer intervals have been shown to be linked with smaller effect sizes (Gershoff et al., 2009). In the current investigation, conducting four measurement waves across nine months was deemed not to place too heavy a burden on school staff and participants, whilst permitting examination of temporal relations across two academic years. 
1 Studies which assess relations between competence and goals within the same PE activity using

2 much shorter time intervals (e.g., 3-4 weeks) may produce larger effect sizes and, in addition,

3 reveal prospective relationships between perceptions of competence and MAp goals (cf.

4 Conroy, Coatsworth, \& Fifer, 2005; Conroy \& Elliot, 2004).

Further work should aim to expand and validate the multidimensional measure of perceived competence, including studies of face validity of existing and new items. Ensuring that each type of competence perception has at least three indicators may offset underidentification problems commonly associated with continuous-time structural equation models (Gershoff et al., 2009). An expanded measure will assist researchers in testing the full model incorporating all types of perceived competence and all achievement goals, as well as testing model invariance across gender. It remains to be established whether the temporal relations between normative competence perceptions and performance goals found in the present study remain significant when the influence of other types of competence and goals is controlled.

The differentiation of types of perceived competence has implications for theory and research in achievement motivation beyond the realm of the current investigation. For example, adopting a self-determination theory perspective (Ryan \& Deci, 2002), how might the informational function of rewards and other external events relate to the satisfaction of competence from mastery, self and normative perspectives? Similarly, physical self-concept researchers may examine the differentiated view of competence to determine relative contribution to individuals’ overall physical self-worth. In summary, the present research provides empirical support for multidimensional definitions of competence, as advocated in the theorizing of Elliot $(1999,2005)$. The prospective influence of competence perceptions on achievement goals was documented, albeit restricted to normative perceived competence and performance goals. These findings indicate 
1 that adopting a more nuanced conceptualization of perceived competence in PE is insightful

2 and we encourage researchers in physical activity to consider a multidimensional perspective in 3 answering their research questions involving the competence construct.

4 
3 school when questionnaires were administered.

4

${ }^{2}$ Details of the CFA and LFI results for each type of perceived competence can be 5 obtained from the first author.

6

${ }^{3}$ Details of the CFA and LFI results for the achievement goals can be obtained from the

7 first author.

8 
Ames, C. (1992). Classrooms: Goals, structures, and student motivation. Journal of Educational Psychology, 84, 261-271. doi: 10.1037/0022-0663.84.3.261

Amiot, C. E., Gaudreau, P., \& Blanchard, C. M. (2004). Self-determination, coping, and goal attainment in sport. Journal of Sport \& Exercise Psychology, 26, 396-411.

Bentler, P. M. (2006). EQS 6 Structural Equations Program Manual. Encino, CA: Multivariate Software Inc.

Bentler, P. M., \& Wu, E. J. C. (2002). EQS 6 for windows: User's guide. Encino, CA: Multivariate Software Inc.

Biddle, S. J. H., Wang, C. K. J., Chatzisarantis, N. L. D., \& Spray, C. M. (2003). Motivation for physical activity in young people: Entity and incremental beliefs about athletic ability. Journal of Sports Sciences, 21, 973-989. doi:10.1080/02640410310001641377

Biddle, S. J. H., Wang, C. K. J., Kavussanu, M., \& Spray, C. M. (2003). Correlates of achievement goal orientations in physical activity: A systematic review of research. European Journal of Sport Science, 3 (5), 1-20. doi:10.1080/17461390300073504

Conroy, D. E., Coatsworth, J. D., \& Fifer, A. M. (2005). Testing dynamic relations between perceived competence and fear of failure in young athletes. Revue Europeene de Psychologie Appliquee/European Review of Applied Psychology, 55, 99-110. doi: 10.1016/j.erap.2004.05.004

Conroy, D. E., \& Elliot, A. J. (2004). Fear of failure and achievement goals in sport: Addressing the issue of the chicken and the egg. Anxiety, Stress and Coping, 17, 271285. doi:10.1080/1061580042000191642

Conroy, D. E., Elliot, A. J., \& Hofer, S. M. (2003). A 2x2 achievement goals questionnaire for sport: Evidence for factorial invariance, temporal stability, and external validity. Journal of Sport \& Exercise Psychology, 25, 456-476. 
1 Conroy, D. E., Kaye, M. P., \& Coatsworth, J. D. (2006). Coaching climates and the destructive effects of mastery-avoidance achievement goals on situational motivation. Journal of Sport \& Exercise Psychology, 28, 69-92.

Conroy, D. E., Metzler, J. N. \& Hofer, S. M. (2003). Factorial invariance and latent mean stability of performance failure appraisals. Structural Equation Modeling, 10, 401-422. doi:10.1207/S15328007SEM1003_4

Covington, M. V. (1992). Making the grade: A self-worth perspective on motivation and school reform. New York: Cambridge University Press.

Dweck, C. S. (1986). Motivational processes affecting learning. American Psychologist, 41, 1040-1048. doi: 10.1037/0003-066X.41.10.1040

Dweck, C. S. (1999). Self theories: Their role in motivation, personality, and development. Philadelphia, PA: Psychology Press.

Elliot, A. J. (1997). Integrating the "classic" and "contemporary" approaches to achievement motivation: A hierarchical model of approach and avoidance achievement motivation. In M. L. Maehr \& P. R. Pintrich (Eds.), Advances in motivation and achievement (Vol. 10, pp. 143-179). Greenwich, CT: JAI Press.

Elliot, A. J. (1999). Approach and avoidance motivation and achievement goals. Educational Psychologist, 34, 169-189. doi:10.1207/s15326985ep3403_3

Elliot, A. J. (2005). A conceptual history of the achievement goal construct. In A. J. Elliot \& C. S. Dweck (Eds.), Handbook of competence and motivation (pp. 55-72). New York: Guildford Press.

Elliot, A. J., \& Church, M. A. (1997). A hierarchical model of approach and avoidance achievement motivation. Journal of Personality and Social Psychology, 72, 218-232. doi: 10.1037/0022-3514.72.1.218 
1 Elliot, A. J., \& Dweck, C. S. (2005). Competence and motivation: Competence as the core of achievement motivation. In A. J. Elliot \& C. S. Dweck (Eds.), Handbook of competence and motivation (pp. 3-12). New York: The Guilford Press.

Elliot, A. J., \& Harackiewicz, J. M. (1996). Approach and avoidance achievement goals and intrinsic motivation: A mediational analysis. Journal of Personality and Social Psychology, 70, 461-475. doi: 10.1037/0022-3514.70.3.461

Elliot, A. J., \& McGregor, H. A. (2001). A 2 x 2 achievement goal framework. Journal of Personality and Social Psychology, 80, 501-519. doi: 10.1037/0022-3514.80.3.501

Elliot, A.J., \& Murayama, K. (2008). On the measurement of achievement goals: Critique, illustration, and application. Journal of Educational Psychology, 100, 613-628. doi: 10.1037/0022-0663.100.3.613

Elliot, A. J., \& Thrash, T. M. (2001). Achievement goals and the hierarchical model of achievement motivation. Educational Psychology Review, 13, 139-156. doi: 10.1023/A:1009057102306

Elliot, A. J., McGregor, H. A., \& Thrash, T. M. (2002). The need for competence. In E. L. Deci \& R. M. Ryan (Eds.), Handbook of self-determination research (pp. 361-387). New York: The University of Rochester Press.

Fleeson, W., \& Noftle, E. E. (2008). Where does personality have its influence? A supermatrix of consistency concepts. Journal of Personality, 76, 1355-1385. doi: 10.1111/j.14676494.2008.00525.x

Gershoff, E. T., Aber, J. L., \& Clements, M. (2009). Parent learning support and child reading ability: A cross-lagged panel analysis for developmental transactions. In A. Sameroff (Ed.), The transactional model of development: How children and contexts shape each other (pp. 203-220). Washington, DC: American Psychological Association. 
1 Goudas, M., Biddle, S., \& Fox, K. (1994). Perceived locus of causality, goal orientations, and perceived competence in school physical education classes. British Journal of Educational Psychology, 64, 453-463.

Hu, L.-T., \& Bentler, P. M. (1999). Cutoff criteria for fit indexes in covariance structure analysis: Conventional criteria versus new alternatives. Structural Equation Modeling, 6, 1-55. doi:10.1080/10705519909540118

Jagacinski, C. M., Kumar, S., Boe, J. L., Lam, H., \& Miller, S. A. (2010). Changes in achievement goals and competence perceptions across the college semester. Motivation and Emotion, 34, 191-204. doi: 10.1007/s11031-010-9165-х

Marsh, H. W., Balla, J. R., \& McDonald, R. P. (1988). Goodness-of-fit indices in confirmatory factor analysis: The effect of sample size. Psychological Bulletin, 103, 391-410. doi: 10.1037/0033-2909.103.3.391

Meredith, W., \& Horn, J. (2001). The role of factorial invariance in modeling growth and change. In L. M. Collins \& A. G. Sayer (Eds.), New methods for the analysis of change (pp. 203-240). Washington, DC: American Psychological Association.

Morris, R. L., \& Kavussanu, M. (2008). Antecedents of approach-avoidance goals in sport. Journal of Sports Sciences, 26, 465-476. doi: 10.1080/02640410701579388

Nicholls, J. G. (1984). Achievement motivation: Conceptions of ability, subjective experience, task choice, and performance. Psychological Review, 91, 328-346. doi:10.1037/0033295X.91.3.328

Nicholls, J. G. (1989). The competitive ethos and democratic education. Cambridge, MA: Harvard University Press.

Office for Standards in Education (2007). Inspection report. Retrieved from http://www.ofsted.gov.uk/oxedu_reports/download/(id)/85039/(as)/121154_302326.pdf 
1 Ryan, R. M., \& Deci, E. L. (2002). Overview of Self-Determination Theory: An organismic 4 dialectical perspective. In E. L. Deci \& R. M. Ryan (Eds.), Handbook of selfdetermination research (pp. 3-33). New York: The University of Rochester Press.

Sameroff, A. (2009). The transactional model. In A. Sameroff (Ed.), The transactional model of development: How children and contexts shape each other (pp. 3-21). Washington, DC: American Psychological Association.

Satorra, A., \& Bentler, P. M. (2001). A scaled difference chi-square test statistic for moment structure analysis. Psychometrika, 66, 507-514. doi: 10.1007/BF02296192

Schutz, R. W. (1998). Assessing the stability of psychological traits and measures. In J. L. Duda (Ed.), Advances in sport and exercise psychology measurement (pp. 393-408). Morgantown, WV: Fitness Information Technology.

Soucy Chartier, I., Gaudreau, P., \& Fecteau, M.-C. (2011). From dispositional affect to academic goal attainment: The mediating role of coping, Anxiety, Stress, \& Coping, 24, 43-58. doi: 10.1080/1061580100372530

Stevenson, S. J., \& Lochbaum, M. R. (2008). Understanding exercise motivation: Examining the revised social-cognitive model of achievement motivation. Journal of Sport Behavior, 31, 389-412.

Wang, C.K.J., Biddle, S.J.H., \& Elliot, A.J. (2007). The 2x2 achievement goal framework in a physical education context. Psychology of Sport and Exercise, 8, 147-168. doi:10.1016/j.psychsport.2005.08.012

Warburton, V.E., \& Spray, C.M. (2008). Motivation in physical education across the primarysecondary school transition. European Physical Education Review, 14, 157-178. doi: 10.1177/1356336X08090704 
Table 1

2 Descriptive Statistics and Internal Consistency Estimates at Each Wave of Measurement

\begin{tabular}{|c|c|c|c|c|c|c|c|c|c|c|c|c|}
\hline \multirow[t]{2}{*}{ Variable } & \multicolumn{3}{|c|}{ Wave 1} & \multicolumn{3}{|c|}{ Wave 2} & \multicolumn{3}{|c|}{ Wave 3} & \multicolumn{3}{|c|}{ Wave 4} \\
\hline & $\mathrm{M}$ & SD & $\alpha$ & $\mathrm{M}$ & SD & $\alpha$ & $\mathrm{M}$ & SD & $\alpha$ & $\mathrm{M}$ & SD & $\alpha$ \\
\hline MAp & 5.02 & 1.39 & .80 & 4.87 & 1.29 & .74 & 4.82 & 1.31 & .76 & 4.52 & 1.33 & .76 \\
\hline PAp & 4.18 & 1.59 & .82 & 4.11 & 1.51 & .84 & 4.13 & 1.46 & .85 & 4.09 & 1.38 & .83 \\
\hline PAv & 4.67 & 1.47 & .72 & 4.36 & 1.50 & .78 & 4.23 & 1.43 & .78 & 4.14 & 1.36 & .79 \\
\hline PC Other & 2.69 & 1.15 & .82 & 2.82 & 1.14 & .78 & 2.82 & 1.09 & .78 & 2.79 & 1.05 & .70 \\
\hline PC Mastery & 3.65 & 0.93 & .79 & 3.63 & 0.86 & .72 & 3.67 & 0.91 & .80 & 3.48 & 0.91 & .76 \\
\hline PC Self & 3.93 & 1.03 & .82 & 3.91 & 0.95 & .75 & 3.90 & 0.93 & .76 & 3.72 & 0.97 & .80 \\
\hline
\end{tabular}

Note MAp = mastery-approach; $\mathrm{PAp}=$ performance-approach; $\mathrm{PAv}=$ performance-avoidance; $\mathrm{PC}=$ perceived competence ${ }^{*} p<.05$ 
1 Table 2

2 Factor Correlations at Each Wave of Measurement

\begin{tabular}{|c|c|c|c|c|c|c|c|c|c|c|c|c|c|c|c|c|c|c|c|c|}
\hline & \multicolumn{4}{|c|}{ МАр } & \multicolumn{4}{|c|}{ PAp } & \multicolumn{4}{|c|}{ PAv } & \multicolumn{4}{|c|}{ PC Other } & \multicolumn{4}{|c|}{ PC Mastery } \\
\hline & W1 & W2 & W3 & W4 & W1 & $\mathrm{W} 2$ & W3 & W4 & $\mathrm{W} 1$ & $\mathrm{~W} 2$ & W3 & W4 & $\mathrm{W} 1$ & W2 & W3 & W4 & W1 & $\mathrm{W} 2$ & W3 & W4 \\
\hline MAp & - & - & - & - & & & & & & & & & & & & & & & & \\
\hline PAp & $.63 *$ & $.61^{*}$ & $.60^{*}$ & $.60 *$ & - & - & - & - & & & & & & & & & & & & \\
\hline PAv & $.64 *$ & $.61^{*}$ & $.55^{*}$ & $.55 *$ & $.88^{*}$ & $.91^{*}$ & $.93^{*}$ & $.95 *$ & - & - & - & - & & & & & & & & \\
\hline PC Other & $.57^{*}$ & $.45^{*}$ & $.56^{*}$ & $.65^{*}$ & $.62 *$ & $.65^{*}$ & $.70^{*}$ & $.72 *$ & $.43^{*}$ & $.49 *$ & $.53 *$ & $.60^{*}$ & - & - & - & - & & & & \\
\hline PC Mastery & $.67^{*}$ & $.78 *$ & $.69 *$ & $.70^{*}$ & $.51^{*}$ & $.50^{*}$ & $.54^{*}$ & $.54^{*}$ & $.40^{*}$ & $.45^{*}$ & $.40 *$ & $.44^{*}$ & $.77^{*}$ & $.69 *$ & $.75^{*}$ & $.80 *$ & - & - & - & - \\
\hline PC Self & $.64^{*}$ & $.66^{*}$ & $.63^{*}$ & $.62 *$ & $.41^{*}$ & $.33^{*}$ & $.31 *$ & $.37 *$ & $.39 *$ & $.32 *$ & $.30 *$ & $.32 *$ & $.55^{*}$ & $.40^{*}$ & $.50 *$ & $.55^{*}$ & $.73 *$ & $.82 *$ & $.77^{*}$ & $.79 *$ \\
\hline
\end{tabular}

Note MAp = mastery-approach; $\mathrm{PAp}=$ performance-approach; $\mathrm{PAv}=$ performance-avoidance; $\mathrm{PC}=$ perceived competence; $\mathrm{W}=$ wave $* p<.05$ 
1 Table 3

2 Temporal Relations Between Multidimensional Perceptions of Competence and Mastery-Approach Goals

\begin{tabular}{|c|c|c|c|c|c|c|c|c|}
\hline Model & $\begin{array}{l}\text { Satorra- } \\
\text { Bentler } \chi^{2}\end{array}$ & $d f$ & $\begin{array}{r}\text { Model } \\
\text { CAIC }\end{array}$ & NNFI & CFI & SRMR & RMSEA & $\begin{array}{l}\text { RMSEA } \\
(90 \% \text { CI) }\end{array}$ \\
\hline \multicolumn{9}{|l|}{ PC Other and MAp goals } \\
\hline Independence & 2547.79 & 205 & & & & & & \\
\hline Full cross-prediction & $331.02 * *$ & 148 & -646.64 & .892 & .922 & .075 & .068 & $.058-.077$ \\
\hline Goals - PC & $337.42 * *$ & 151 & -660.06 & .892 & .920 & .081 & .067 & $.058-.077$ \\
\hline PC - Goals & $331.90 * *$ & 151 & -665.58 & .895 & .923 & .074 & .066 & $.057-.076$ \\
\hline No cross-prediction & $338.01 * *$ & 154 & -679.28 & .895 & .921 & .080 & .066 & $.057-.076$ \\
\hline Model comparisons & $\Delta \mathrm{SB} \chi^{2}$ & $\Delta d f$ & & & & & & \\
\hline Full cross-prediction vs. Goals - PC & 6.37 & 3 & & & & & & \\
\hline Full cross-prediction vs. PC - Goals & 2.13 & 3 & & & & & & \\
\hline Goals - PC vs. No cross-prediction & 1.93 & 3 & & & & & & \\
\hline PC - Goals vs. No cross-prediction & 6.07 & 3 & & & & & & \\
\hline \multicolumn{9}{|l|}{$\begin{array}{l}\text { PC Mastery and MAp goals } \\
\end{array}$} \\
\hline Independence & 2120.35 & 205 & & & & & & \\
\hline Full cross-prediction & $229.00 * *$ & 148 & -748.66 & .941 & .958 & .072 & .045 & $.033-.056$ \\
\hline Goals - PC & $232.34 * *$ & 151 & -765.13 & .942 & .958 & .073 & .045 & $.033-.055$ \\
\hline PC - Goals & $232.11 * *$ & 151 & -765.37 & .943 & .958 & .073 & .045 & $.033-.055$ \\
\hline No cross-prediction & $235.15^{* *}$ & 154 & -782.14 & .944 & .958 & .075 & .044 & $.032-.055$ \\
\hline Model comparisons & $\Delta \mathrm{SB} \chi^{2}$ & $\Delta d f$ & & & & & & \\
\hline Full cross-prediction vs. Goals - PC & 3.32 & 3 & & & & & & \\
\hline Full cross-prediction vs. PC - Goals & 2.84 & 3 & & & & & & \\
\hline Goals - PC vs. No cross-prediction & 2.49 & 3 & & & & & & \\
\hline PC - Goals vs. No cross-prediction & 3.02 & 3 & & & & & & \\
\hline \multicolumn{9}{|l|}{ PC Self and MAp goals } \\
\hline Independence & 2060.31 & 205 & & & & & & \\
\hline Full cross-prediction & $226.51 * *$ & 148 & -751.15 & .941 & .958 & .073 & .044 & $.032-.055$ \\
\hline Goals - PC & $228.65 * *$ & 151 & -768.93 & .943 & .958 & .071 & .044 & $.032-.055$ \\
\hline PC - Goals & $231.28 * *$ & 151 & -766.20 & .941 & .957 & .074 & .044 & $.032-.055$ \\
\hline No cross-prediction & $233.09 * *$ & 154 & -784.21 & .943 & .957 & .072 & .044 & $.032-.054$ \\
\hline Model comparisons & $\Delta \mathrm{SB} \chi^{2}$ & $\Delta d f$ & & & & & & \\
\hline Full cross-prediction vs. Goals - PC & 1.97 & 3 & & & & & & \\
\hline Full cross-prediction vs. PC - Goals & 4.75 & 3 & & & & & & \\
\hline Goals - PC vs. No cross-prediction & 4.46 & 3 & & & & & & \\
\hline PC - Goals vs. No cross-prediction & 1.76 & 3 & & & & & & \\
\hline
\end{tabular}

3 Note. MAp = mastery-approach; $\mathrm{PC}$ = perceived competence

$4 \quad * * p<.01$ 
1 Table 4

2 Temporal Relations Between Multidimensional Perceptions of Competence and Performance-Approach Goals

\begin{tabular}{|c|c|c|c|c|c|c|c|c|}
\hline Model & $\begin{array}{l}\text { Satorra- } \\
\text { Bentler } \chi^{2}\end{array}$ & $d f$ & $\begin{array}{l}\text { Model } \\
\text { CAIC }\end{array}$ & NNFI & CFI & SRMR & RMSEA & $\begin{array}{l}\text { RMSEA } \\
(90 \% \text { CI })\end{array}$ \\
\hline \multicolumn{9}{|l|}{ PC Other and PAp goals } \\
\hline Independence & 3123.68 & 205 & & & & & & \\
\hline Full cross-prediction & $185.54 *$ & 148 & -792.12 & .982 & .987 & .050 & .031 & $.013-.044$ \\
\hline Goals - PC & $205.80 * *$ & 151 & -791.68 & .975 & .981 & .086 & .037 & $.023-.048$ \\
\hline PC - Goals & $187.48 *$ & 151 & -810.00 & .983 & .988 & .055 & .030 & $.012-.043$ \\
\hline No cross-prediction & $206.78 * *$ & 154 & -810.52 & .976 & .982 & .088 & .036 & $.021-.047$ \\
\hline Model comparisons & $\Delta \mathrm{SB} \chi^{2}$ & $\Delta d f$ & & & & & & \\
\hline Full cross-prediction vs. Goals - PC & $26.63 *$ & 3 & & & & & & \\
\hline Full cross-prediction vs. PC - Goals & 1.46 & 3 & & & & & & \\
\hline PC - Goals vs. No cross-prediction & $24.62 *$ & 3 & & & & & & \\
\hline \multicolumn{9}{|l|}{ PC Mastery and PAp goals } \\
\hline Independence & 2581.28 & 205 & & & & & & \\
\hline Full cross-prediction & 176.05 & 148 & -801.61 & .984 & .988 & .055 & .026 & $.000-.040$ \\
\hline Goals - PC & $181.94 *$ & 151 & -815.53 & .982 & .987 & .065 & .027 & $.005-.041$ \\
\hline PC - Goals & 176.92 & 151 & -820.56 & .985 & .989 & .058 & .025 & $.000-.039$ \\
\hline No cross-prediction & $183.84 *$ & 154 & -833.45 & .983 & .987 & .071 & .027 & $.000-.040$ \\
\hline Model comparisons & $\Delta \mathrm{SB} \chi^{2}$ & $\Delta d f$ & & & & & & \\
\hline Full cross-prediction vs. Goals - PC & 5.78 & 3 & & & & & & \\
\hline Full cross-prediction vs. PC - Goals & 0.80 & 3 & & & & & & \\
\hline Goals - PC vs. No cross-prediction & 1.76 & 3 & & & & & & \\
\hline PC - Goals vs. No cross-prediction & 6.92 & 3 & & & & & & \\
\hline \multicolumn{9}{|l|}{ PC Self and PAp goals } \\
\hline Independence & 2553.15 & 205 & & & & & & \\
\hline Full cross-prediction & $211.15^{* *}$ & 148 & -766.28 & .963 & .973 & .061 & .040 & $.027-.051$ \\
\hline Goals - PC & $214.35^{* *}$ & 151 & -783.12 & .963 & .973 & .062 & .039 & $.026-.051$ \\
\hline PC - Goals & $213.86 * *$ & 151 & -786.62 & .964 & .973 & .063 & .039 & $.026-.051$ \\
\hline No cross-prediction & $216.81 * *$ & 154 & -800.49 & .964 & .973 & .064 & .039 & $.026-.050$ \\
\hline Model comparisons & $\Delta \mathrm{SB} \chi^{2}$ & $\Delta d f$ & & & & & & \\
\hline Full cross-prediction vs. Goals - PC & 2.79 & 3 & & & & & & \\
\hline Full cross-prediction vs. PC - Goals & 2.18 & 3 & & & & & & \\
\hline Goals - PC vs. No cross-prediction & 2.02 & 3 & & & & & & \\
\hline PC - Goals vs. No cross-prediction & 2.57 & 3 & & & & & & \\
\hline
\end{tabular}

3 Note. $\mathrm{PAp}=$ performance-approach; $\mathrm{PC}=$ perceived competence

$* p<.05 * * p<.01$ 
1 Table 5

2 Temporal Relations Between Multidimensional Perceptions of Competence and Performance-Avoidance Goals

\begin{tabular}{|c|c|c|c|c|c|c|c|c|}
\hline Model & $\begin{array}{l}\text { Satorra- } \\
\text { Bentler } \chi^{2}\end{array}$ & $d f$ & $\begin{array}{r}\text { Model } \\
\text { CAIC }\end{array}$ & NNFI & CFI & SRMR & RMSEA & $\begin{array}{l}\text { RMSEA } \\
(90 \% \text { CI })\end{array}$ \\
\hline \multicolumn{9}{|l|}{ PC Other and PAv goals } \\
\hline Independence & 2541.24 & 205 & & & & & & \\
\hline Full cross-prediction & $237.18^{* *}$ & 148 & -740.48 & .947 & .962 & .053 & .047 & $.036-.058$ \\
\hline Goals - PC & $249.21 * *$ & 151 & -748.27 & .943 & .958 & .073 & .049 & $.038-.059$ \\
\hline PC - Goals & $241.49 * *$ & 151 & -755.98 & .947 & .961 & .052 & .047 & $.036-.058$ \\
\hline No cross-prediction & $253.00 * *$ & 154 & -764.29 & .944 & .958 & .071 & .049 & $.038-.059$ \\
\hline Model comparisons & $\Delta \mathrm{SB} \chi^{2}$ & $\Delta d f$ & & & & & & \\
\hline Full cross-prediction vs. Goals - PC & $12.92 *$ & 3 & & & & & & \\
\hline Full cross-prediction vs. PC - Goals & 4.17 & 3 & & & & & & \\
\hline PC - Goals vs. No cross-prediction & $12.57 *$ & 3 & & & & & & \\
\hline \multicolumn{9}{|l|}{ PC Mastery and PAv goals } \\
\hline Independence & 2059.50 & 205 & & & & & & \\
\hline Full cross-prediction & 189.89* & 148 & -787.77 & .969 & .977 & .058 & .032 & $.016-.045$ \\
\hline Goals - PC & 193.54* & 151 & -803.93 & .969 & .977 & .061 & .032 & $.016-.045$ \\
\hline PC - Goals & $191.81 *$ & 151 & -805.67 & .970 & .978 & .056 & .032 & $.015-.044$ \\
\hline No cross-prediction & $195.14^{*}$ & 154 & -822.15 & .970 & .978 & .059 & .031 & $.015-.044$ \\
\hline Model comparisons & $\Delta \mathrm{SB} \chi^{2}$ & $\Delta d f$ & & & & & & \\
\hline Full cross-prediction vs. Goals - PC & 3.62 & 3 & & & & & & \\
\hline Full cross-prediction vs. PC - Goals & 1.80 & 3 & & & & & & \\
\hline Goals - PC vs. No cross-prediction & 1.50 & 3 & & & & & & \\
\hline PC - Goals vs. No cross-prediction & 3.27 & 3 & & & & & & \\
\hline \multicolumn{9}{|l|}{ PC Self and PAv goals } \\
\hline Independence & 2029.73 & 205 & & & & & & \\
\hline Full cross-prediction & $200.99 * *$ & 148 & -776.67 & .960 & .971 & .056 & .036 & $.022-.048$ \\
\hline Goals - PC & $204.04 * *$ & 151 & -793.43 & .961 & .971 & .057 & .036 & $.022-.048$ \\
\hline PC - Goals & $205.61^{* *}$ & 151 & -791.87 & .959 & .970 & .061 & .037 & $.023-.048$ \\
\hline No cross-prediction & $208.53 * *$ & 154 & -808.77 & .960 & .970 & .061 & .036 & $.022-.048$ \\
\hline Model comparisons & $\Delta \mathrm{SB} \chi^{2}$ & $\Delta d f$ & & & & & & \\
\hline Full cross-prediction vs. Goals - PC & 2.84 & 3 & & & & & & \\
\hline Full cross-prediction vs. PC - Goals & 4.67 & 3 & & & & & & \\
\hline Goals - PC vs. No cross-prediction & 4.53 & 3 & & & & & & \\
\hline PC - Goals vs. No cross-prediction & 2.68 & 3 & & & & & & \\
\hline
\end{tabular}

3 Note. $\mathrm{PAv}=$ performance-avoidance; $\mathrm{PC}=$ perceived competence

$4 \quad{ }^{*} p<.05 * * p<.01$ 
Figure 1

Prospective relations between perceived competence and achievement goals tested in the present study.

a) No cross-prediction model

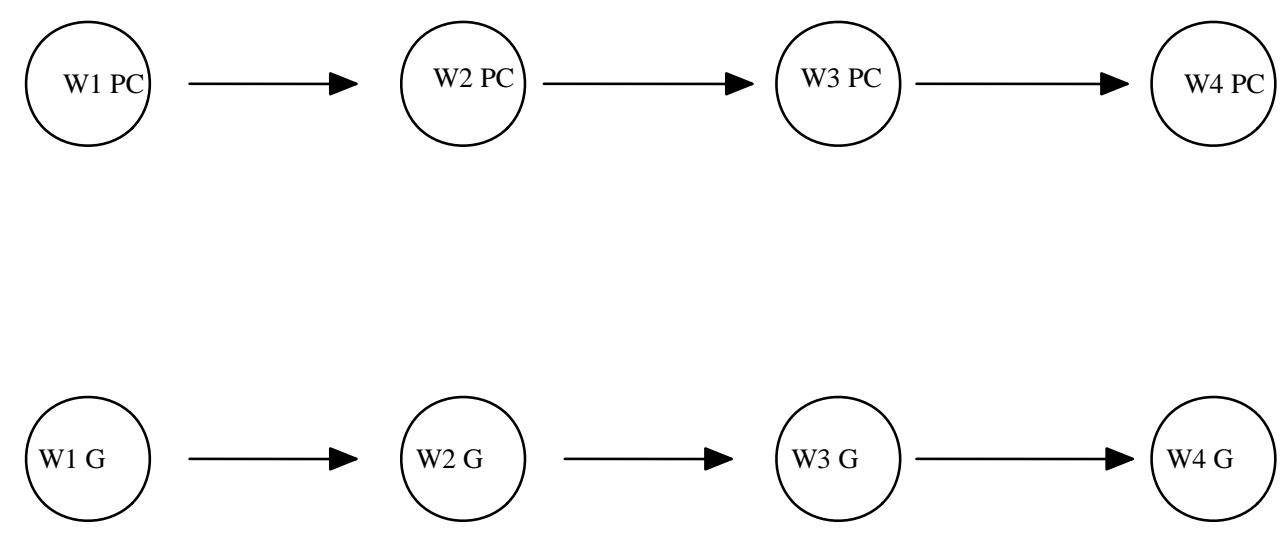

b) Goals - PC model

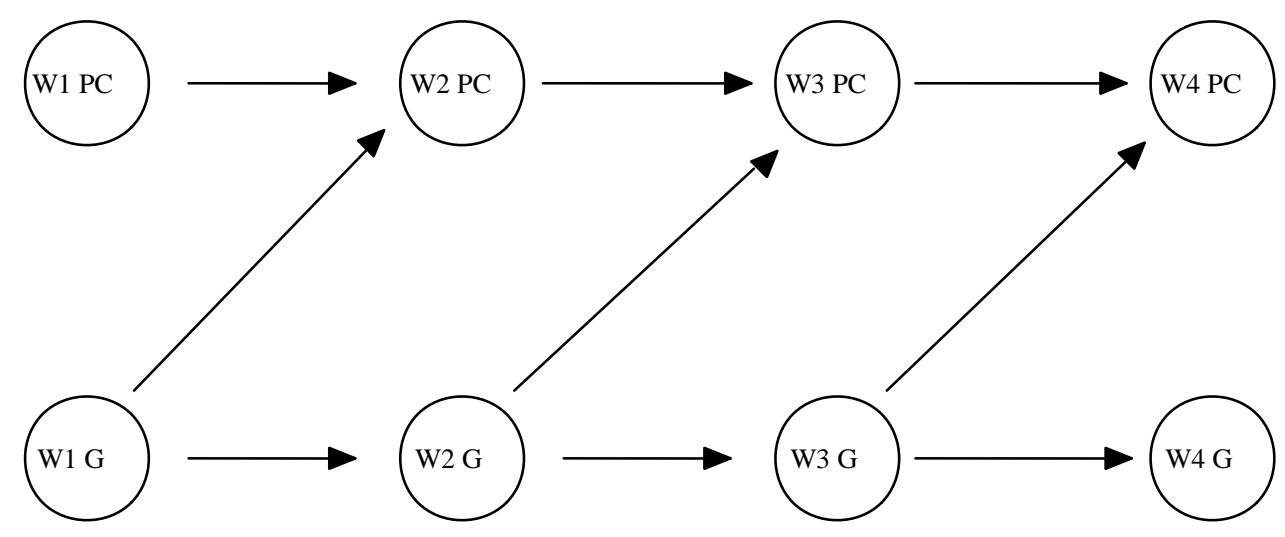

c) PC-goals model

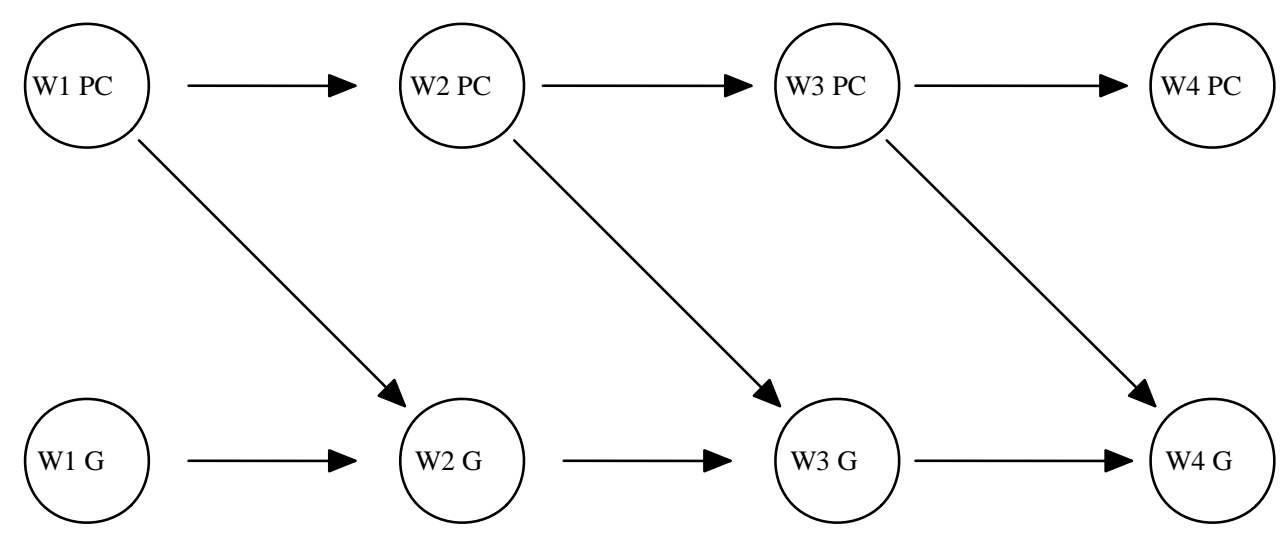


d) Full cross-prediction model

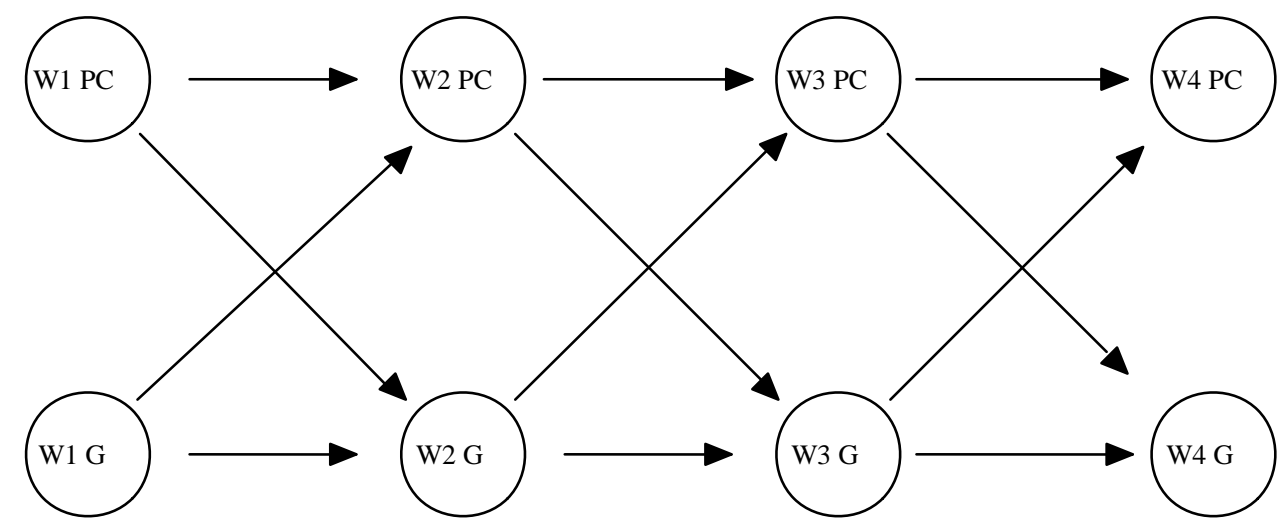

Note. Measurement model parameters and within time correlated factors (wave [W] 1) and disturbances (waves 2, 3 and 4) are omitted for the sake of clarity. PC = type of perceived competence; $\mathrm{G}$ = type of achievement goal. 
Figure 2

Prospective relations between PC Other (PC O) and performance-approach (PAp) goals.

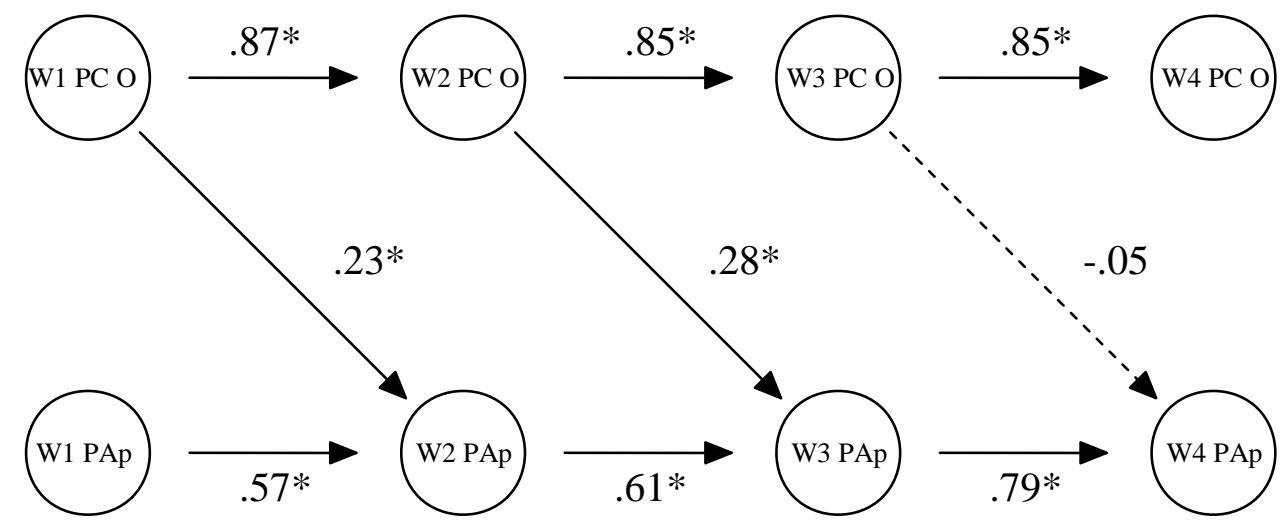

Note. Measurement model parameters and within time correlated factors (wave [W] 1) and disturbances (waves 2, 3 and 4) are omitted for the sake of clarity. $* p<.05$ 
Figure 3

Prospective relations between PC Other (PC O) and performance-avoidance (PAv) goals.

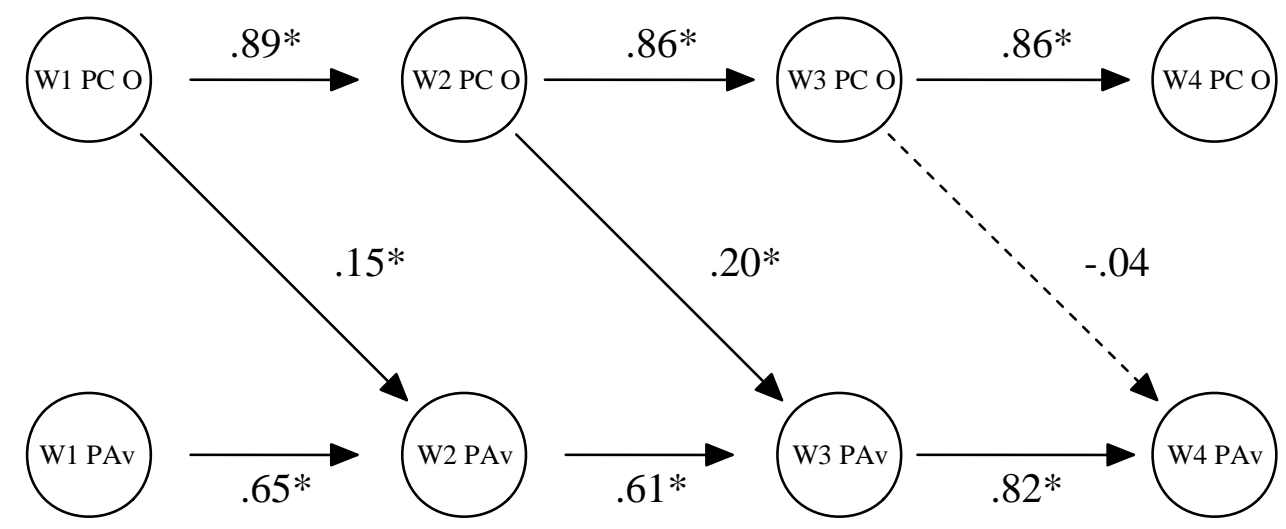

Note. Measurement model parameters and within time correlated factors (wave [W] 1) and disturbances (waves 2, 3 and 4) are omitted for the sake of clarity. $* p<.05$ 